\title{
AluMine: alignment-free method for the discovery of polymorphic Alu element insertions
}

\author{
Tarmo Puurand, Viktoria Kukuškina, Fanny-Dhelia Pajuste and Maido Remm ${ }^{*}$
}

\begin{abstract}
Background: Recently, alignment-free sequence analysis methods have gained popularity in the field of personal genomics. These methods are based on counting frequencies of short $k$-mer sequences, thus allowing faster and more robust analysis compared to traditional alignment-based methods.

Results: We have created a fast alignment-free method, AluMine, to analyze polymorphic insertions of Alu elements in the human genome. We tested the method on 2,241 individuals from the Estonian Genome Project and identified 28,962 potential polymorphic Alu element insertions. Each tested individual had on average 1,574 Alu element insertions that were different from those in the reference genome. In addition, we propose an alignment-free genotyping method that uses the frequency of insertion/deletion-specific 32-mer pairs to call the genotype directly from raw sequencing reads. Using this method, the concordance between the predicted and experimentally observed genotypes was $98.7 \%$. The running time of the discovery pipeline is approximately $2 \mathrm{~h}$ per individual. The genotyping of potential polymorphic insertions takes between 0.4 and $4 \mathrm{~h}$ per individual, depending on the hardware configuration.
\end{abstract}

Conclusions: AluMine provides tools that allow discovery of novel Alu element insertions and/or genotyping of known Alu element insertions from personal genomes within few hours.

Keywords: Alu repeat element, Mobile element insertions, Alignment-free sequence analysis

\section{Introduction}

More than $55 \%$ of the human genome contains repeated sequences [1-4]. These repeated sequences can be divided into tandem repeats and interspersed repeat elements (segmental duplications and transposable elements). The most abundant transposable element in the human genome is the Alu element. A typical Alu element is an approximately $300 \mathrm{bp}$ long transposable nucleotide sequence [5-7]. The estimated number of full-length or partial Alu elements in the human genome is 1.1 million [8-11].

The presence or absence of some Alu elements is variable between individual genomes. Many Alu elements actively retrotranspose themselves into new locations, thus generating polymorphic Alu insertions [12-14]. A polymorphic Alu in this context refers to the presence

\footnotetext{
* Correspondence: maido.remm@ut.ee

Institute of Molecular and Cell Biology, University of Tartu, Tartu, Estonia
}

or absence of the entire element and not single nucleotide polymorphisms within the Alu sequence. The insertion rate of Alu elements into new locations is approximately one insertion per 20 births $[15,16]$. Polymorphic insertions of mobile DNA elements can disrupt coding regions, reprogram chromatin methylation patterns or disturb the regulation of flanking genes [17-21]. These changes in the genome can lead to disease [22-25]. Therefore, computational methods that reliably detect polymorphic Alu element insertions from sequencing data are needed.

Several methods for the identification of polymorphic Alu insertions have been developed that include the following: VariationHunter [26, 27], Hydra [28], TEA [29], RetroSeq [30], alu-detect [31] and Tangram [32], MELT [33], T-lex2 [34], STEAK [35], me-scan [36] and unnamed method used for analysing HGDP data [37]. All these methods are based on the mapping of sequencing reads and the subsequent interpretation of mapping 
results. The discovery of new insertions is typically based on split locations of a single read and/or the distance between paired reads.

Several databases or datasets that describe polymorphic Alu insertions are available. The oldest resource containing known polymorphic transposable elements is the dbRIP database [38]. It contains insertions detected by comparison of Human Genome Project data with Celera genome data. dbRIP also contains information about somatic Alu insertions that might be related to different diseases. The most comprehensive Alu element dataset is available from the 1000 Genome Project (1000G) [16, 33]. Phase 3 of the 1000G project studied 2504 individuals. They identified 1,236 Alu elements absent from the reference genome and 12,748 Alu elements inserted to the reference genome [33]. A subset of these sequences has been validated by Sanger sequencing [13].

We have developed a set of novel, alignment-free methods for the rapid discovery of polymorphic Alu insertions from fully sequenced individual genomes. In addition, we provide a method that calls genotypes with previously known insertions directly from raw reads. Evaluation of these methods was performed by computational simulations and PCR product size analysis.

\section{Results}

\section{Rationale for the alignment-free discovery of Alu} insertion sites

We describe a novel method allowing both the discovery of new polymorphic Alu insertions and the detection of known insertions directly from raw reads in next generation sequencing (NGS) data. Two key steps within the discovery method are the a) identification of potential polymorphic Alu insertions present in tested personal genomes but not in the reference genome (REF- discovery) and the b) identification of potential polymorphic Alu elements present in the current reference genome (REF+ discovery) that might be missing in the tested genomes.

All discovery pipelines use a $10 \mathrm{bp}$ consensus sequence from the $5^{\prime}$ end of the Alu (GGCCGGGCGC) to detect Alu elements from sequences. The consensus sequence is complemented with all possible sequences containing the same consensus wit one nucleotide change (one mismatch). We refer to this set of 31 sequences as Alu signature sequences (see Additional file 2: Table S1). It is important to realize that this approach relies on intact 5 -end of the element and it is not able to detect any 5 ' truncated elements. The REF- discovery pipeline identifies all occurrences of Alu signatures in raw sequencing reads from an individual. A $25 \mathrm{bp}$ flanking sequence from the $5^{\prime}$ region is recorded together with the discovered Alu signature sequence (Additional file 1: Figure S1). Subsequently, the location of these $25 \mathrm{bp}$ sequences in the reference genome is determined using the custom-made software gtester (Kaplinski, unpublished). A new REF- element is reported if the $10 \mathrm{bp}$ sequence in the raw reads is different from the $10 \mathrm{bp}$ sequence in the reference genome.

The REF+ discovery pipeline uses the same set of Alu element signatures to identify all locations in the reference genome where the preceding $5 \mathrm{bp}$ target site duplication motif (TSD) is present $270-350 \mathrm{bp}$ downstream from the signature sequence (see Additional file 1: Figure S2 for details). Both discovery pipelines generate a pair of 32-mers for each identified Alu element (Fig. 1). Initially used 35 -mers $(25+10)$ are shortened to 32-mers

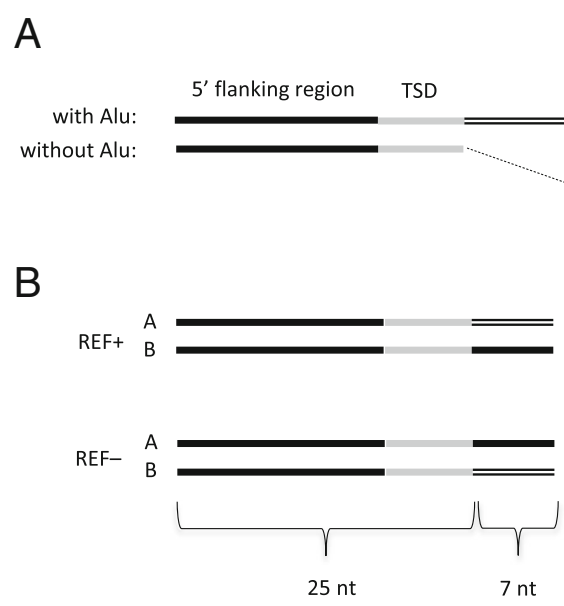

Fig. 1 Principle of creating k-mer pairs for the calling (genotyping) of polymorphic Alu element insertions. a Genomic regions with or without an Alu element. b A pair of 32-mers is created from the insertion breakpoint region covering 25 nucleotides from the $5^{\prime}$-flanking region and 7 nucleotides from either the Alu element or the $3^{\prime}$-flanking region. Allele A always represents the sequence from the reference genome and allele $B$ represents the alternative, non-reference allele 
at this step because we use the $k$-mer managing software package GenomeTester4, which is able to handle $k$-mers with a maximum length of 32 nucleotides. Two 32-mers in a pair correspond to two possible alleles with or without the Alu element insertion. See the section Parameter choice in discussion for additional explanations of chosen $k$-mer lengths.

The principles of the generation of $k$-mer pairs specific to Alu insertion breakpoints are shown in Fig. 1. To detect polymorphic insertions, we use $25 \mathrm{bp}$ from the reference genome immediate to the $5^{\prime}$ end of the potential Alu insertion point and then add either $7 \mathrm{bp}$ from the Alu element or $7 \mathrm{bp}$ from the genomic sequence downstream of the second TSD motif (Fig. 1a). All candidate 32-mer pairs are further filtered based on their genotypes in test individuals.

The alignment-free genotyping of known Alu elements is based on counting the frequencies of 32-mer pairs specific to Alu element breakpoints using the previously published FastGT software package [39]. The names of two alleles are assigned based on their status in the reference genome; the allele that is present in the reference genome is always called allele $\mathrm{A}$, and the alternative allele is always called allele B (Fig. 1b). This allows us to use the same naming convention for alleles and genotypes used by the FastGT package for single nucleotide variants. The entire discovery process is outlined in Fig. 2. These 32-mer pairs are used for the subsequent genotyping of the Alu elements in other individuals.

\section{Compilation of the list of potential polymorphic Alu elements}

To test the applicability of the AluMine method to real data, we performed REF- element discovery using 2,241 high-coverage genomes from the Estonian Genome Project [40] and compiled a set of 32-mer pairs for subsequent genotyping. REF- candidates consist of Alu

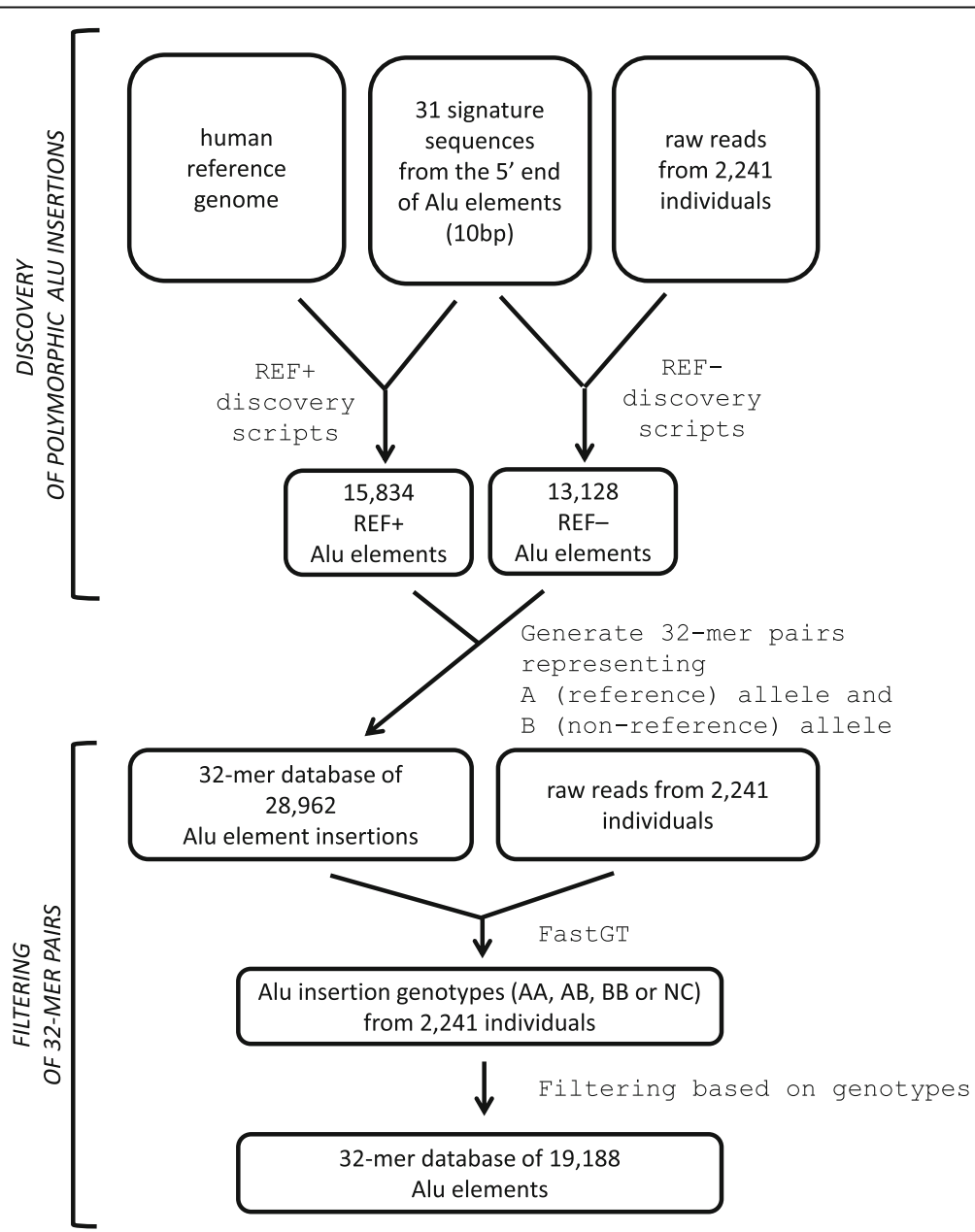

Fig. 2 Overview of the discovery methods. Potential polymorphic Alu elements were identified from the raw reads of high-coverage WGS data (REF- Alu elements) and the reference genome (REF+ Alu elements). The candidate Alu elements were filtered using a subset of high-coverage individuals. A final set of 32-mers was used for the fast calling of polymorphic insertions from raw sequencing reads 
elements that are present in the raw reads from sequenced individuals but not in the reference genome. We searched the raw reads from test individuals following the principles described above and detected 13,128 REFAlu elements overall.

REF+ discovery was performed using the human reference genome version 37. We searched for potential REF+ candidates by using the following criteria: the element must have an intact Alu signature sequence, have a TSD at least $5 \mathrm{bp}$ long on both ends of the Alu element, have more than 100 bits similar to known Alu elements, and must not be present in the chimpanzee genome. Our REF+ script detected 267,377 elements with an Alu signature sequence from the human reference genome. However, only 15,834 (5.9\%) of these passed all the above-mentioned filtering criteria and remained in the set of potential polymorphic elements. We do not assume or claim that all of these REF+ elements are polymorphic. The elements that are 100\% monomorphic in Estonian population can still be polymorphic in other populations. We selected a larger set in purpose, so people can use all these potential elements in studies involving personal genomes from other populations. The proportion of different signature sequences among the set of REF+ elements is shown in Additional file 2: Table S1. All the steps involved in Alu element discovery are summarized in Table 1 together with the number of elements that passed each step.

\section{Simulation tests of the discovery method}

We realize that although our discovery methods detected more than 13,000 REF- Alu element insertions,

Table 1 Number of REF- and REF+ candidates after different filtering steps

\begin{tabular}{|c|c|}
\hline \multicolumn{2}{|l|}{ REF- filtering steps } \\
\hline REF- variations detected in 2,241 individuals & 572,081 \\
\hline $\begin{array}{l}\text { REF- candidates that can be located in the reference } \\
\text { genome }\end{array}$ & 379,523 \\
\hline $\begin{array}{l}\text { REF- candidates that have unique location in the reference } \\
\text { genome }\end{array}$ & 298,907 \\
\hline $\begin{array}{l}\text { REF- candidates after removal of duplicate, closely located } \\
\text { and GC-rich k-mers }\end{array}$ & 13,128 \\
\hline REF- elements that generate reliable genotypes & 9,712 \\
\hline \multicolumn{2}{|l|}{ REF+ filtering steps } \\
\hline Alu signature sequences detected in the reference genome & 267,377 \\
\hline REF+ candidates with $5 \mathrm{bp}$ TSD sequence within $270-350 \mathrm{bp}$ & 110,938 \\
\hline REF+ candidates with BLAST homology & 98,711 \\
\hline $\begin{array}{l}\text { REF+ candidates that are not present in chimpanzee } \\
\text { genome }\end{array}$ & 16,434 \\
\hline REF+ candidates after removal of duplicate k-mers & 15,834 \\
\hline REF+ candidates that generate reliable genotypes & 13,396 \\
\hline
\end{tabular}

some polymorphic Alu elements remain undiscovered in given individuals. There are two obvious reasons why Alu variants are missed in the REF- discovery step: a) a low depth of coverage in some individuals and b) difficulties with the unique localization of 25 -mers in some genomic regions.

The effect of coverage on the discovery rate can be estimated from simulated data. We generated data with $5 \times$ to $55 \times$ nucleotide-level coverage and analyzed how many REF- elements we would discover from these with our method. The results are shown in Fig. 3a. There is an association between the depth of coverage and the discovery rate, which levels out at an approximately $40 \times$ depth of coverage.

Another factor affecting the sensitivity of Alu element discovery is that the repeated structure of the genome sequence prevents the unique localization of discovered Alu elements. The REF- discovery method relies on the unique localization of the 25-mer in front of the Alu signature sequence. We decided to perform a series of simulations with artificial Alu element insertions to determine what fraction of them was discoverable by our REF- discovery method. For this, we inserted 1,000 typical Alu elements into random locations of a diploid genome sequence and generated random sequencing reads from this simulated genome using wgsim software [41]. The simulation was repeated with 10 male and 10 female genomes using different mutation rates. Varying the mutation rate helps to somewhat simulate older and younger Alu element insertions (older Alu elements have accumulated more mutations) and estimate how their detection rate varies accordingly. We observed that 20 to $23 \%$ of the elements remain undetected, depending on the mutation rate (Fig. 3b). The mutation rate has only a moderate effect on the sensitivity of detection; thus, we assume that the age of the Alu element insertion does not significantly influence the number of detected elements. Additionally, $7 \%$ of the inserted elements remained undiscovered because they were inserted into regions with unknown sequence, containing long stretches of N's. This number is independent of mutation rate. Unsequenced regions of the genome remain inaccessible to any Alu element discovery method that is based on sequencing.

\section{Comparison with other Alu discovery methods}

When comparing the results of Alu discovery methods, we can compare two aspects. If the same individuals are studied by many methods, we can estimate the overlap between identified elements. Otherwise, we can compare the overall number of detected elements.

We were able to identify the overlap between Alu elements discovered from sample NA12878 within the $1000 \mathrm{G}$ pilot project and the 1000G Phase3 project. The 


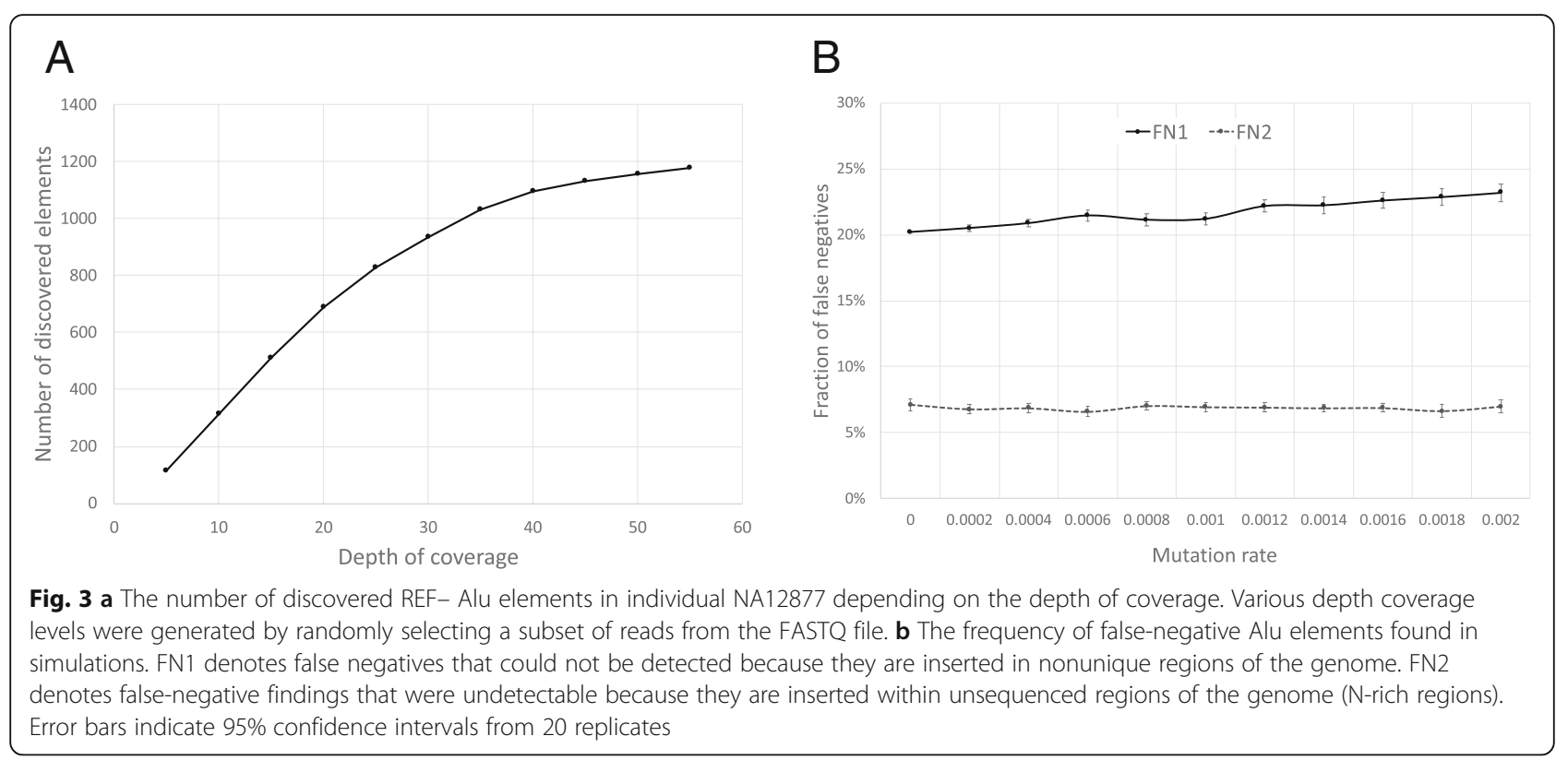

overlaps between methods are similar for REF+ and REF- elements. AluMine discovered $63 \%$ of elements reported by the $1000 \mathrm{G}$ Pilot Phase in the sample NA12878 plus an additional 458 elements (Fig. 4). Three hundred sixty elements reported by $1000 \mathrm{G}$ Pilot Phase remained undiscovered by AluMine. Our preliminary analysis indicates that at least $221(61 \%)$ of these undiscovered elements are shorter than expected full length Alu element. We assume that these are mostly 5 '-truncated elements that AluMine cannot discover with the current algorithm. Additional reasons for missing REFelements are non-unique 25-mer in front of the element (9\% of missed cases), SNV within 25-mer (8\% of missed cases) and atypical Alu signature sequence (7\% of missed cases).
To examine other methods, we were only able to compare the overall number of discovered REF- elements. AluMine detected 1,116 and 1,127 REF- insertions in the CEPH individuals NA12877 and NA12878 and 1,290 insertions in NA18506. alu-detect discovered on average 1,339 Alu insertions per CEU individual [31]. Hormozdiari et al. detected 1,282 events in the CEU individual NA10851 with $22 \times$ coverage and 1,720 events in the YRI individual NA18506 with 40× coverage [26]. TEA detected an average of 791 Alu insertions in each individual genome derived from cancer samples [29]. In genomes from Chinese individuals, $\mathrm{Yu}$ et al. discovered 1,111 Alu element insertions on average [43]. Thus, the overall number of detected REF- elements was similar for all methods.

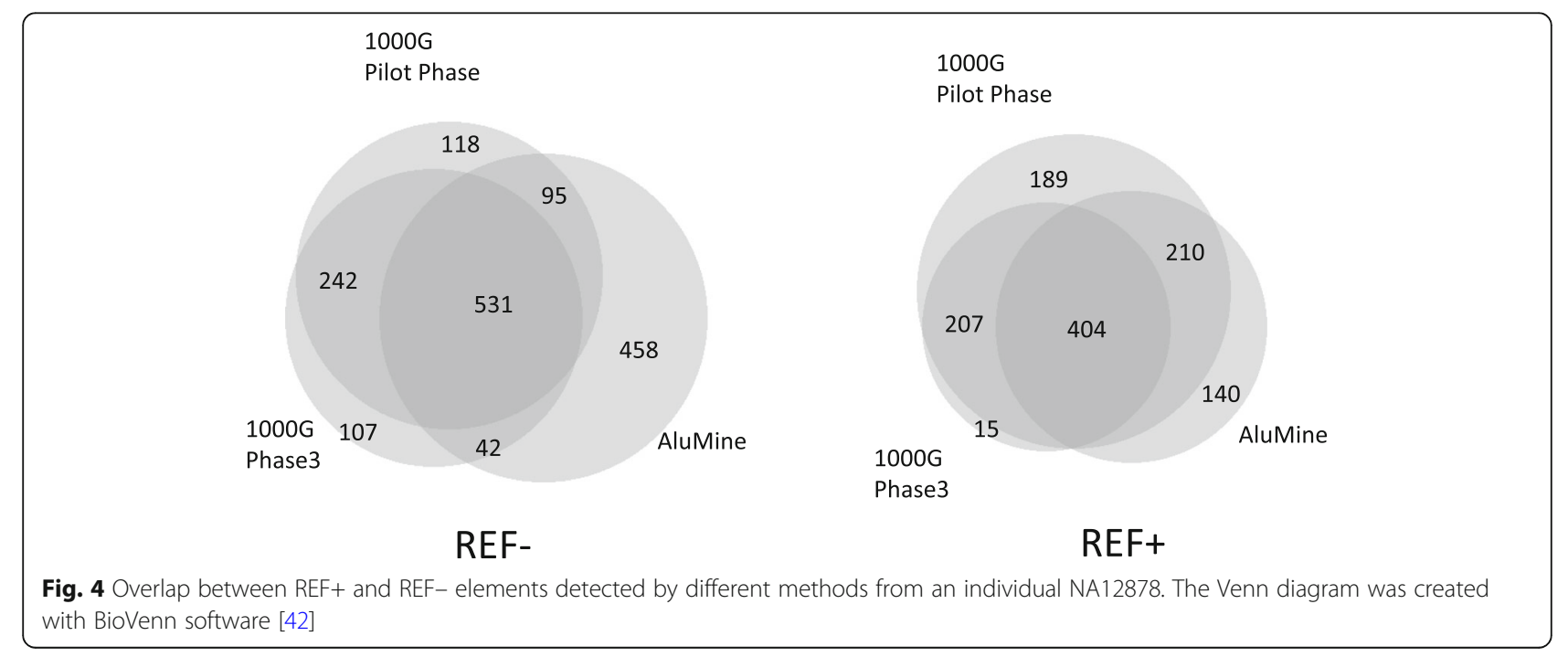




\section{Frequency of non-reference Alu elements in tested individuals}

We scanned 2,241 Estonian individuals with the final filtered set of Alu elements to identify the genotypes of all potential polymorphic Alu insertions in their genomes. All tested individuals had some Alu elements that were different from those in the reference genome. The tested individuals had 741-1,323 REF- elements (median 1, 045) that were not present in the reference genome and 465-651 REF+ Alu elements (median 588) that were present in the reference genome but missing in given individual (Fig. 5).

One interesting question that can be addressed from the given data is the cumulative number of REF- elements in a population. We discovered 14,455 REF- Alu elements from 2,241 tested individuals. However, many of these were common within the population. Thus, saturation of the total number of polymorphic elements is expected if sufficient number of individuals are sequenced. The saturation rate of the REF- elements is shown in Fig. 6. Obviously, the number of REF- elements was still far from saturation. Each new individual genome sequence still contained 2-3 previously unseen REF- elements.

\section{Selection of 32-mers for genotyping}

In principle, we would like to call the genotypes with discovered Alu elements in other individuals using pairs of specific 32-mers and FastGT genotyping software. Unfortunately, not all discovered Alu elements are suitable for fast genotyping with a pair of short k-mers. Some of them tend to give excessive counts from other regions of the genome, and some might be affected by common Single Nucleotide Variants (SNVs). To select a set of Alu elements that gives reliable genotype calls, we filtered the Alu elements based on their genotyping results using data from the same 2,241 individuals that were used for REF- element discovery. For this, we merged 32-mers of REF- and REF+ Alu elements with a set of SNVspecific 32-mers and determined the genotypes of these markers in test individuals using the FastGT package. SNV-specific $k$-mers are required at this step because Alu elements alone cannot provide reliable estimates of parameter values for the empirical Bayes classifier used in FastGT. Additional filtering and removal of candidate elements was based on several criteria. We removed elements that generated an excessive number of unexpected genotypes (a diploid genotype is expected for autosomes, and a haploid genotype is expected for chrY), elements that deviated from Hardy-Weinberg equilibrium and monomorphic REF- elements. The validation of all tested markers together with their genotype counts is shown in Additional file 2: Table S2. In the final validated $k$-mer database, we included 9,712 polymorphic REF- elements that passed the validation filters, including 1,762 polymorphic REF+ elements and 11,634 monomorphic REF+ elements.

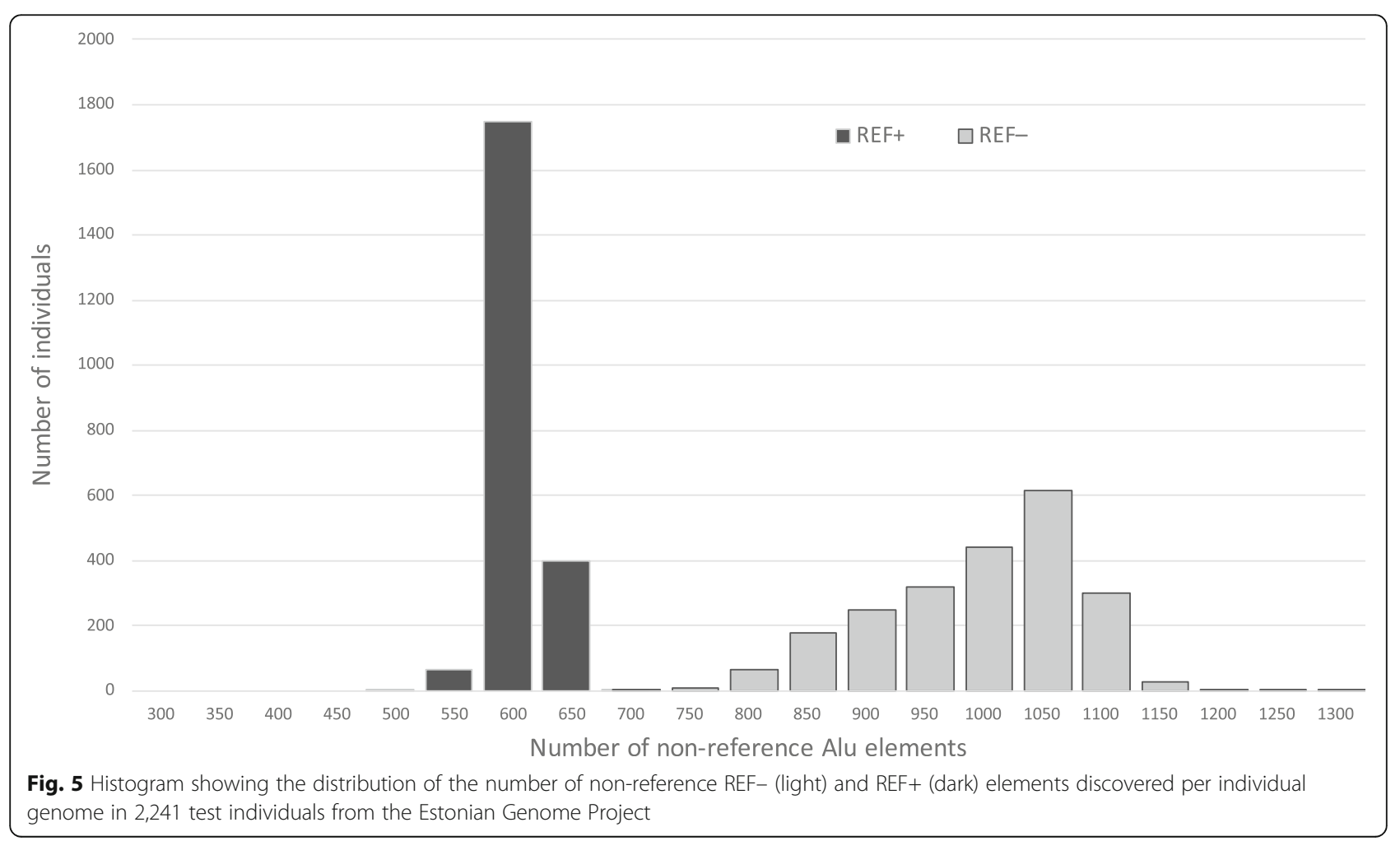




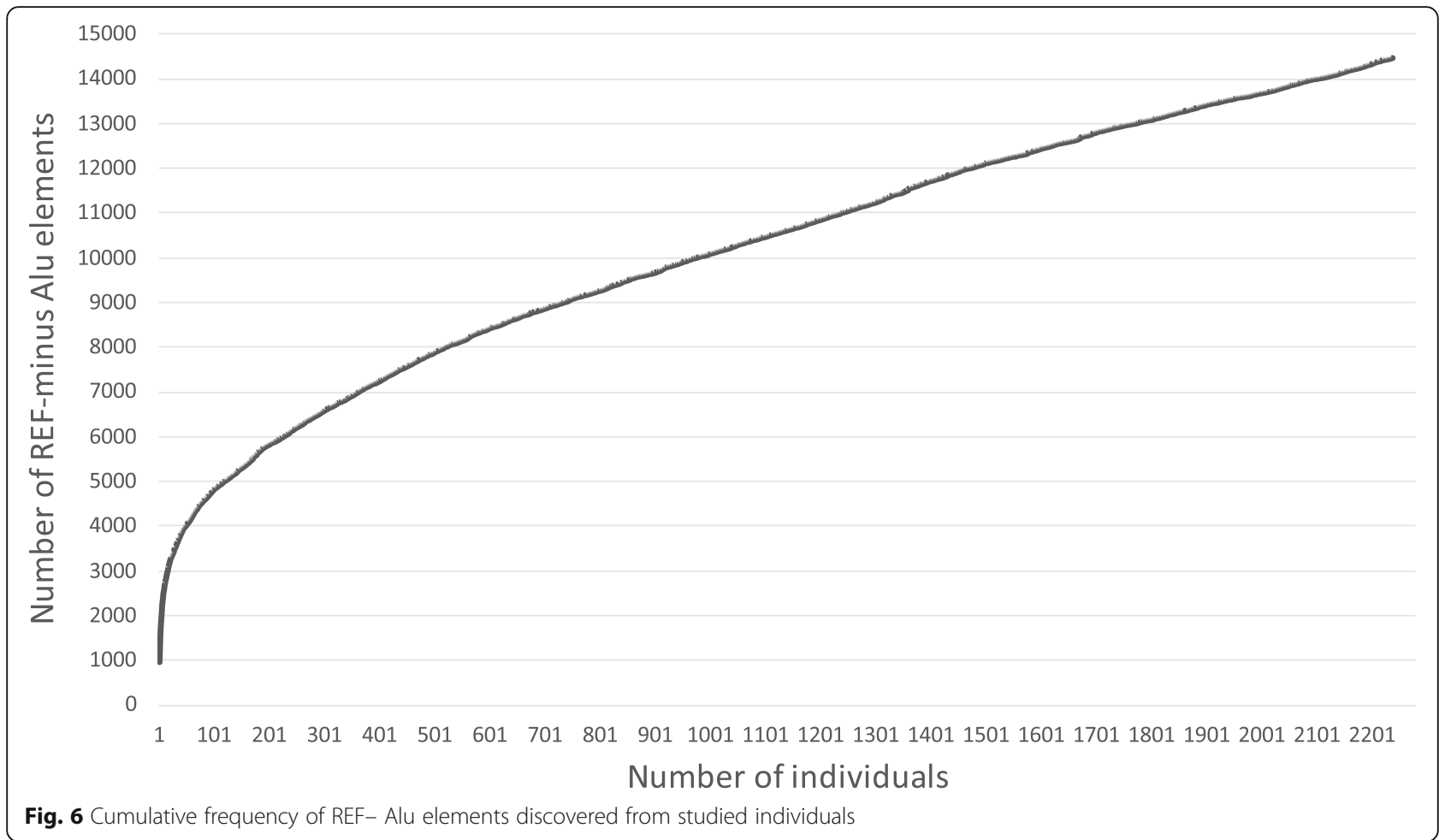

Although $87 \%$ of the candidate REF+ elements were monomorphic in the tested individuals, the possibility exists that they are polymorphic in other populations; therefore, we did not remove them from the $k$-mer database.

\section{Validation of the discovery by using family data}

Additional validation approach is based on mendelian inheritance errors in CEPH family \#1463. We tested the number of mendelian inheritance errors of discovered REF- Alu elements in two father-mother-child trios with founders (grandparents). All REF- discoveries in child were considered. We verified whether at least one of the parents had discovery in the same location. This way 2, 662 discoveries were tested and 87 of these were with mendelian inheritance conflict. Thus the observed False Discovery Rate (FDR) in this dataset is $3.3 \%$. It should be kept in mind that trio analysis is not able to detect all errors, and therefore the actual FDR can be slightly larger. Full list of inheritance patterns of these REF- elements are shown in Additional file 2: Table S3.

\section{Experimental validation}

We decided to validate the alignment-free genotyping of polymorphic Alu elements with a subset of newly discovered Alu elements. The validation was performed experimentally using PCR fragment length polymorphism. We used four different Alu elements (1 REF- and 3 REF+ elements) and determined their genotypes in 61 individuals. The individuals used in this validation did not belong to the training set of 2,241 individuals and were sequenced independently. The electrophoretic gel showing the PCR products of one REF- polymorphism is shown in Fig. 7. The results for the three REF+ individuals are shown in Fig. 8. The computationally predicted genotypes and experimentally determined genotypes conflicted in only 3 cases; thus, the concordance rate was $98.7 \%$. The 32 -mer counts, predicted genotypes and experimental genotypes for each individual are shown in Additional file 2: Table S4.

However, this validation approach was based only on 4 discovered elements and demonstrates the accuracy of genotyping rather than accuracy of Alu element discovery. To estimate the False Discovery Rate (FDR) of REFelement discovery we performed another PCR experiment with more REF- elements as suggested by reviewers of this manuscript. The selection of elements for validation and PCR primer design is described in Methods. We tested 48 REF- elements in three individuals that did not belong to the training set and were not used for the selection of candidate elements (Additional file 1: Figure S4). In these individuals we had overall 68 predicted REF- elements, 4 of which turned out to be false predictions (6\% FDR). This is slightly higher than an estimate from family trios, but this is expected because family analysis cannot detect all existing errors. These markers were discovered and tested on a different set of individuals. This could potentially cause underestimation of the FDR among rare elements that were 


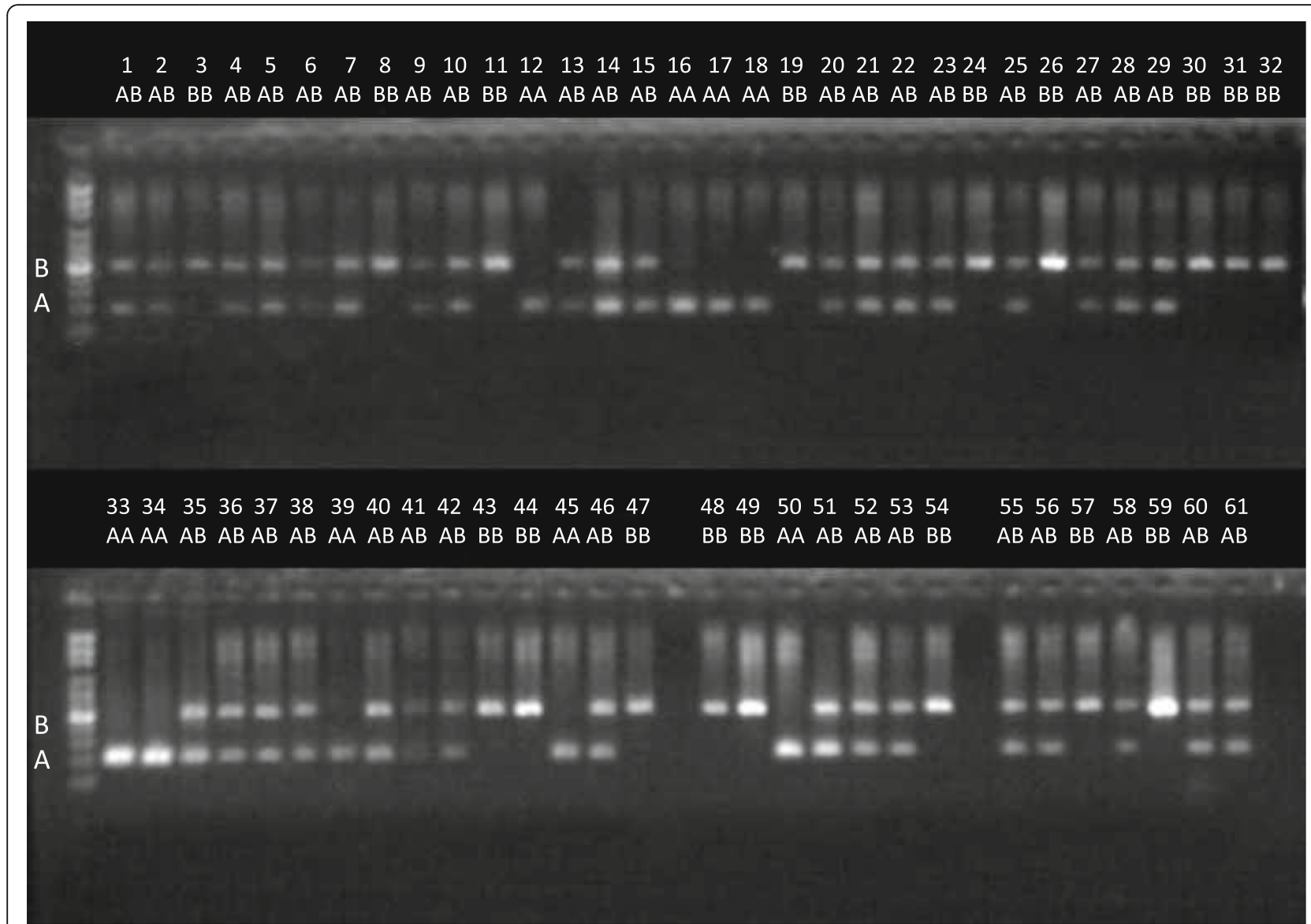

Fig. 7 A gel electrophoretic image showing the experimental validation of polymorphic Alu element insertion (REF- elements). One polymorphic Alu element from chr8:42039896 was tested by PCR in DNA from 61 individuals. Lower bands show the absence of an Alu insertion (reference allele A), and upper bands show its presence (alternative allele B)

discovered only in a single individual (singletons). On the other hand, trio analysis, described in the previous paragraph, should show much higher FDR if such underestimation for singletons would exist. As we observed rather low mendelian error rate in family trios, we are confident that FDR for low frequency elements is not considerably higher than observed for validated elements. Predicted and observed genotypes for each primer pair and each individual are shown in Additional file 2: Table S5.

\section{Performance}

The performance of the AluMine methods can be divided into three parts: the performance of the REFdiscovery pipeline, the performance of the REF+ discovery pipeline and the genotyping performance. The REF+ pipeline was run on a server with a $2.27 \mathrm{GHz}$ Intel Xeon CPU X7560 and 512 GB RAM. The REF- scripts and genotyping were run on cluster nodes with a $2.20 \mathrm{GHz}$ Intel Xeon CPU E5-2660 and 64 GB RAM.

The most time-consuming steps in the REF- discovery pipeline are a) searching for Alu signatures from FASTQ files, which takes $2 \mathrm{~h}$ per individual on a single CPU core, and b) finding their locations in the reference genome using gtester software ( $2 \mathrm{~h}$ for the first individual, $4 \mathrm{~min}$ for each subsequent individual). The increase in speed for subsequent individuals is due to the large size of the gtester indices (approximately $60 \mathrm{~GB}$ ). For the first individual, they are read from a hard drive, and for subsequent individuals, the disk cache is used. None of the steps require more than $8 \mathrm{~GB}$ of RAM.

The REF+ discovery pipeline contains the following three time-consuming steps: a) a search for 31 different Alu signatures from chromosomes of the reference genome (takes $14 \mathrm{~min}), \mathrm{b}$ ) a homology search with all the candidates to confirm that they are Alu elements ( $2 \mathrm{~min}$ ) and c) a comparison with the chimpanzee genome to exclude fixed Alu elements ( $4 \mathrm{~min}, 28 \mathrm{~GB}$ RAM). All these steps use a single processor. The REF+ discovery pipeline has to be run only once and should not be repeated for each separate individual. Thus, in terms of performance, it occupies only a minor part of the overall analysis. 


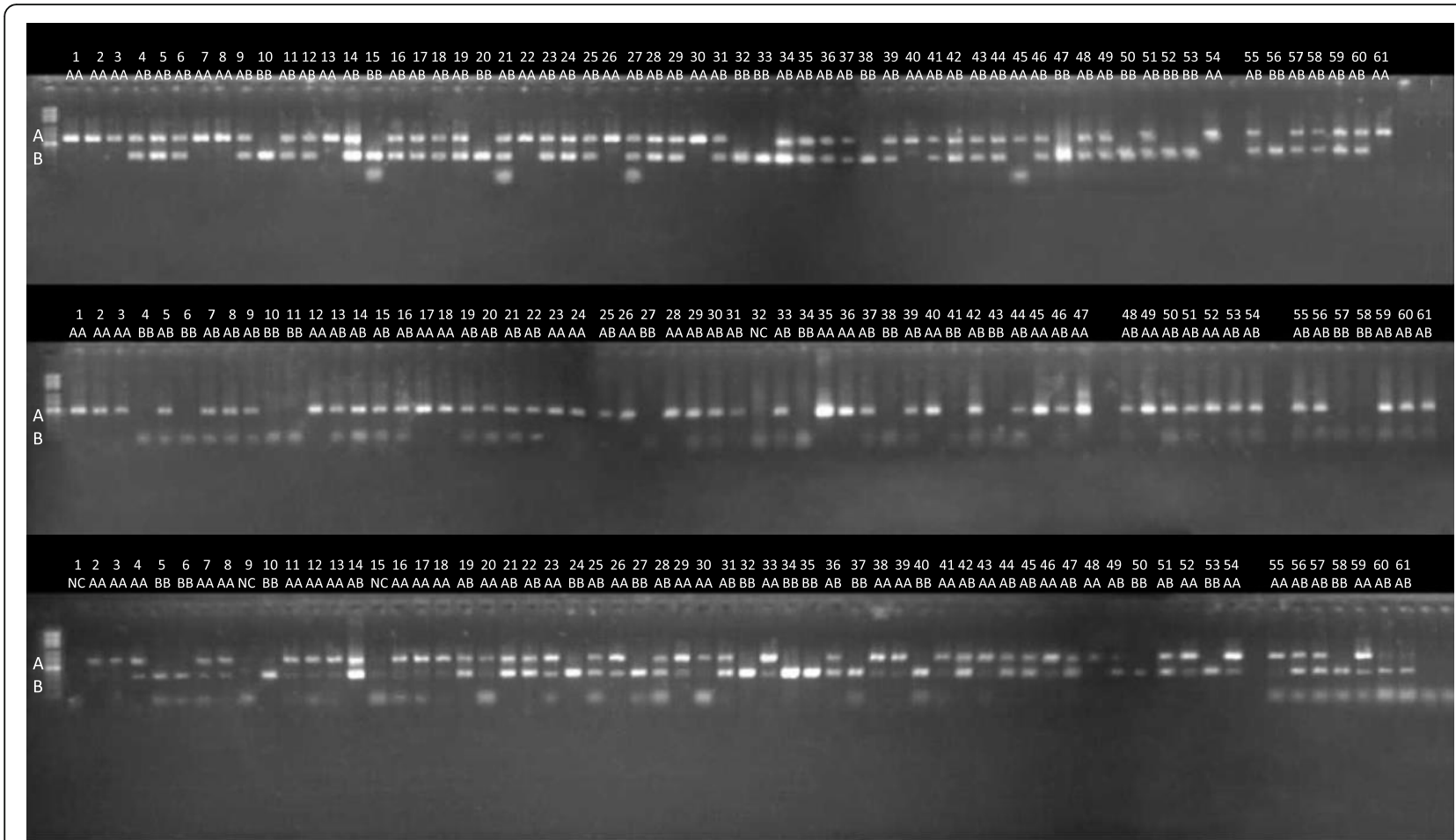

Fig. 8 A gel electrophoretic image showing the experimental validation of REF+ polymorphic Alu element insertions. Three locations from chr1:169160349, chr15:69049897 and chr3:95116523 were tested by PCR in DNA from 61 individuals. Upper bands show the presence of an Alu insertion (reference allele A), and lower bands show its absence (alternative allele B)

The genotyping of individuals is performed with the previously published FastGT package [39]. The performance of FastGT was analyzed in the original paper. In optimized conditions (> $200 \mathrm{~GB}$ RAM available, using FASTQ instead of BAM format, and using solid state drive), it can process one high coverage individual within 30 min. However, we used FastGT on cluster nodes with a limited amount of hard drive space and limited RAM. Therefore, in our settings, FastGT acquired sequence data from BAM files through standard input, which limited its performance. In this way, we were able to process one individual in 3-4 CPU hours.

\section{Discussion}

\section{Parameter choice}

A common matter of discussion for alignment-free sequence analysis methods is the optimal length of $k$-mers. In our case, the $k$-mers used for genotyping Alu elements had to be bipartite and contain sufficient sequence from the genome and a couple of nucleotides from the Alu element (Fig. 2). The first part of the bipartite $k$-mer must guarantee the unique localization of the $k$-mer in the human genome; the second part must allow distinguishing variants with and without the Alu element at a given location. Both parts must fit into 32 nucleotides because we use the $k$-mer managing software package GenomeTester4, which is able to handle $k$-mers with a maximum length of 32 nucleotides. In the current work, we chose to divide 32 -mers into $25+7$ nucleotides. Our previous work demonstrated that all $k$-mers 22 to 32 nucleotides long should perform equally well to analyze variations in the human genome (Fig. 5 in [39]). Thus, we assume that we would obtain a rather similar genotyping result with slightly different splits, such as $22+10,23+9$ or $24+8$ nucleotides. Using fewer than 7 nucleotides from the Alu element would give too high of a chance to have an identical sequence in the reference genome, and the program would not be able to distinguish variants with and without Alu. Current pipeline of REF- discovery is optimized for personal genomes with $20 \mathrm{x}$ to $40 \mathrm{x}$ coverage. Using it on data with very high or very low coverage might need tweaking of parameters. For example, due to algorithmic reasons, the minimum and maximum frequency of potential REF- elements is hardcoded in the script, not adjusted dynamically based on depth of coverage. These parameters can be changed in AluMine/discovery_REF-minus/find_ref_minus_candidates_bam.pl, line 39. Depth of coverage for tested individuals is shown in Additional file 1: Figure S3.

\section{Comparison with other software}

We compared the number of REF- elements discovered by different methods. However, the direct comparison of 
these numbers to our data is complicated because different populations and individuals were used in different reports. The number of discovered insertions was correlated with the individual ancestry of the subjects: generally, fewer Alu insertions were discovered in CEU individuals than in YRI individuals [16]. Additionally, the depth of coverage had a strong effect on the results, as shown in Fig. 3a. All methods, including AluMine, detected approximately 1000 REF- elements per genome. The slight differences were likely due to differences in the depth of coverage and the different origins of the samples used.

Different detection methods have different biases. The premature termination of target primed reverse transcription during the replication of Alu elements can generate truncated Alu element insertions that are missing the $5^{\prime}$ end of the element. It has been estimated that $16.4 \%$ of Alu elements are truncated insertions [37]. Furthermore, some Alu element polymorphisms appear through the deletion of existing elements (2\%) [13] or mechanisms that do not involve retrotransposition (less than 1\%) [37]. Our REF+ method relies on the presence of TSDs, and the REF- method relies on the presence of intact $5^{\prime}$ ends in the Alu. Thus, we would not be able to detect those events, which would explain the majority of the differences between our results and the elements detected in the 1000G pilot phase (Fig. 4).

\section{The number of REF+ elements}

We identified 15,834 potentially polymorphic REF+ elements, of which 1,762 were polymorphic in at least one individual in the studied population. The number of polymorphic REF+ elements (present in the reference genome) has been studied less thoroughly. The number of human-specific Alu insertions has been reported to be 8,817 [4], thus our number might seem unreasonable.

We extracted all 270-350 bp long regions that have TSD and significant homology to known Alu elements, so they are certainly Alu elements. However, we cannot guarantee that all of them are human-specific. Only very robust comparison with chimp genome is performed during the discovery. It is possible to do more scrutinized manual analysis of these candidate elements. More careful homology search with chimpanzee (and perhaps bonobo) genomes might reveal that some or many of these REF+ elements are not human specific.

On the other hand, we do not focus here on finding the actual number of human-specific elements, but rather on the method for discovering and genotyping these potentially human-specific elements. The method is relatively fast and having some additional elements in the dataset would not compromise the speed of genotyping nor interpretation of the results. The elements that are not poymorphic or not human-specific would show up as AA genotypes in all tested individuals and should not interfere with subsequent analyses. It should not be a problem if some of these are not really polymorphic or even not human-specific. We believe that it is better to provide more candidates, so people can use them in large-scale population-based genotyping studies. One just has to keep in mind that the list provided by us is a list of candidate elements, not the final list of validated human-specific Alu elements.

\section{Future directions}

In principle, our discovery method can be used to search for novel Alu elements in any whole-genome sequencing data. Transposable elements are known to occur in genes that are commonly mutated in cancer and to disrupt the expression of target genes [22, 29]. Our method allows the discovery of novel Alu elements from sequences from tumors and matched normal blood samples, allowing the study of the somatic insertion of Alu elements in cancer cells and their role in tumorigenesis. The precompiled set of 32-mer pairs allows the genotyping of known Alu element insertions in high-coverage sequencing data. This facilitates the use of Alu elements in genome-wide association studies along with SNVs.

The alignment-free discovery method could also be adapted for the detection of other transposable elements, such as L1 or SVA elements. However, the discovery of these elements is more complicated because SVA elements contain a variable number of $(\text { CCCTCT })_{n}$ repeats in their $5^{\prime}$ end, and L1 elements contain variable number of Gs in front of the GAGGAGCCAA signature sequence. These difficulties can be solved by allowing variable length between element's signature sequence and 25-mer from the reference genome.

\section{Conclusions}

We have created a fast, alignment-free method, AluMine, to analyze polymorphic insertions of Alu elements in the human genome. It consists of two pipelines for the discovery of novel polymorphic insertions directly from raw sequencing reads. One discovery pipeline searches for Alu elements that are present in a given individual but missing from the reference genome (REF- elements), and the other searches for potential polymorphic Alu elements present in the reference genome but missing in some individuals (REF+ elements). We applied the REF- discovery method to 2,241 individuals from the Estonian population and identified 13,128 polymorphic REF- elements overall. We also analyzed the reference genome and identified 15,834 potential polymorphic REF+ elements. Each tested individual had on average 1,574 Alu element insertions (1,045 REFand $588 \mathrm{REF}+$ elements) that were different from those in the reference genome. 
In addition, we propose an alignment-free genotyping method that uses the frequency of insertion/deletion-specific 32-mer pairs to call the genotype directly from raw sequencing reads. We tested the accuracy of the genotyping method experimentally using a PCR fragment length polymorphism assay. The concordance between the predicted and experimentally observed genotypes was $98.7 \%$.

The running time of the REF- discovery pipeline is approximately $2 \mathrm{~h}$ per individual, and the running time of the REF+ discovery pipeline is $20 \mathrm{~min}$. The genotyping of potential polymorphic insertions takes between 0.4 and $4 \mathrm{~h}$ per individual, depending on the hardware configuration.

\section{Methods and data}

\section{Genome data}

The reference genome GRCh37.p13 was used for all analyses.

\section{Discovery of REF- and REF+ elements}

The exact details of all discovery pipelines are described in the corresponding scripts (pipeline_ref_plus.sh, pipeline_ref_minus.sh and pipeline_merging_and_filtering.sh) available from GitHub (https://github.com/bioinfo-ut/ AluMine). The scripts are written in BASH and PERL. FASTA files, $k$-mer databases and files with coordinates of all discovered Alu elements are downloadable from http://bioinfo.ut.ee/?page_id=167\&lang=en.

\section{Validation of Alu elements by PCR}

One hundred PCR primer pairs were designed to amplify randomly selected Alu elements discovered from two sequenced individuals (V000985a and V51287) from the Estonian Genome Project (EGP) panel. The PCR primers were designed using Primer3 software package [44, 45], using SNP masking and repeat masking options $[46,47]$. Repeat masking option was used to reject all candidate primers which had masked region within $4 \mathrm{bp}$ from 3 '-end. First 48 primer pairs from this set of primers were used for validation experiments shown in Additional file 1: Figure S4 and in Additional file 2: Table S5. The chromosomal coordinates of the elements selected for PCR validation and their allele frequencies in population are shown in Additional file 2: Table S5 and Table S6. The PCR experiments were performed on 61 (Figs. 7 and 8) or 3 (Additional file 1: Figure S4) independently sequenced individuals from another project. Genome sequence data of test individuals was not used neither for training of AluMine nor for selection of the candidate elements.

\section{PCR protocol}

To prepare a $20 \mu$ PCR master mix, we mixed $0.2 \mu$ FIREPol DNA polymerase (Solis BioDyne, Estonia), $0.6 \mu \mathrm{l}$ of
$10 \mathrm{mM}$ DNTP, $0.8 \mu \mathrm{l}$ of a $20 \mathrm{mM}$ primer mix, $2 \mu \mathrm{l}$ of 25 $\mathrm{mM} \mathrm{MgCl} 2,2 \mu \mathrm{l}$ polymerase buffer, and $14.4 \mu \mathrm{l}$ Milli-Q water. For PCR, Applied Biosystems thermocyclers were used. The PCR was run for 30 cycles using a 1 min denaturation step at $95^{\circ} \mathrm{C}$, a $1 \mathrm{~min}$ annealing step at $55^{\circ} \mathrm{C}$ and a $1.5 \mathrm{~min}$ elongation step at $72^{\circ} \mathrm{C}$. For gel electrophoresis, a $1.5 \%$ agarose gel $(0.5 \mathrm{mM} \mathrm{TBE}+$ agarose tablets + EtBr) was used. The PCR primer pairs used for the amplification of potential polymorphic regions are shown in Additional file 2: Table S6.

\section{Simulated Alu insertions}

To simulate polymorphic Alu insertions, we inserted 1000 heterozygous Alu elements into random locations of the diploid reference genome together with a $15 \mathrm{bp}$ target site duplication sequence and a random length polyA sequence $(5-80 \mathrm{bp})$. A male genome (5.98 Gbp) and a female genome $(6.07 \mathrm{Gbp})$ were generated by merging two copies of autosomal chromosomes and the appropriate number of sex chromosomes into a single FASTA file. Simulated sequencing reads were generated using wgSim (version 0.3.1-r13) software from the SAMtools package [41]. The following parameters were used: haplotype_mode $=1$, base_error_rate $=0.005$, outer_distance_between_the_two_ends $=500$, length_of_reads $=$ 151 , cutoff_for_ambiguous_nucleotides $=1.0$, and number_of_reads $=306,000,000$.

\section{Additional files}

\section{Additional file 1: Figure S1 and Figure S2 explaining the REF- and REF+ discovery algorithms. Figure S3 showing distribution of depth of coverage in tested individuals. Figure $\mathbf{5 4}$. A gel electrophoretic image showing the experimental validation of REF- polymorphic Alu element insertions in 48 locations and three individuals. Upper bands show the presence of an Alu insertion (alternative allele B), and lower bands show its absence (reference allele A). Predicted genotypes and expected product lengths are shown in Additional file 2: Table S5. PCR primers used for this analysis are shown in Additional file 2: Table S6. (DOCX 501 kb) \\ Additional file 2: Table S1 showing frequencies of Alu signature sequences. Table S2 showing filtering statistics based on genotyping of EGP individuals. Table S3 showing results of REF- element discovery in two CEPH families. Table S4 and Table S5 summarizing results of PCR validation. TableS6 showing PCR primer sequences and PCR product lengths. (XLSX 2079 kb)}

\section{Abbreviations}

1000G: 1000 Genome Project; NGS: Next Generation Sequencing; REF- Alu element: Polymorphic Alu element present in at least one personal genome but not in the reference genome; REF+ Alu element: Polymorphic Alu element present in the reference genome, but missing in at least one personal genome; SNV: Single Nucleotide Variant; TSD: Target Site Duplication motif

\section{Acknowledgements}

The authors thank Kadri Maal for help with the PCR experiments and Lauris Kaplinski for advice on improving performance of Alu element discovery algorithms and for adapting the $k$-mer counting software FastGT and gtester for this project. 


\section{Authors' contributions}

TP conceived the idea and performed most of the large-scale genomic analyses. MR wrote the scripts for post-processing of the data, performed simulations and wrote the manuscript. VK performed all the PCR experiments and helped to develop genome analysis methods. FDP provided help with data management and visualization. All authors read and approved the final manuscript.

\section{Funding}

This work was funded by institutional grant IUT34-11 from the Estonian Research Council and the EU ERDF grant No. 2014-2020.4.01.15-0012 (Estonian Center of Excellence in Genomics and Translational Medicine). The cost of the sequencing of individuals from the Estonian Genome Center was partly covered by the Broad Institute (MA, USA) and the PerMed I project from the TERVE program. Computation was partly carried out in the High Performance Computing Center of the University of Tartu.

\section{Availability of data and materials}

All scripts (pipeline_ref_plus.sh, pipeline_ref_minus.sh and pipeline_merging_and_filtering.sh) and software (gtester) created for this study are available from GitHub (https://github.com/bioinfo-ut/AluMine). The FastGT package used for genotyping the Alu insertions is also available from GitHub (https://github.com/bioinfo-ut/GenomeTester4/blob/master/README. FastGT.md). K-mer lists for genotyping Alu elements using FastGT are available from University of Tartu webpage (http://bioinfo.ut.ee/FastGT/). FASTA files and $k$-mer databases with discovered Alu elements are downloadable from http:// bioinfo.ut.ee/?page_id=167\&lang=en. The whole genome sequencing data that support the findings of this study are available on request from Estonian Genome Centre (https://www.geenivaramu.ee/en) but restrictions apply to the availability of these data, and so are not publicly available.

\section{Ethics approval and consent to participate}

The genome data were collected and used with ethical approval (Nr. 206 T4, obtained for the project SP1GVARENG).

\section{Consent for publication}

Not applicable.

\section{Competing interests}

The authors declare that they have no competing interests.

\section{Received: 21 March 2019 Accepted: 12 July 2019}

\section{Published online: 18 July 2019}

\section{References}

1. de Koning APJ, Gu W, Castoe TA, Batzer MA, Pollock DD. Repetitive elements may comprise over two-thirds of the human genome. PLoS Genet. 2011;7:e1002384.

2. Wheeler TJ, Clements J, Eddy SR, Hubley R, Jones TA, Jurka J, et al. Dfam: a database of repetitive DNA based on profile hidden Markov models. Nucleic Acids Res. 2013:41:D70-82.

3. Hubley R, Finn RD, Clements J, Eddy SR, Jones TA, Bao W, et al. The Dfam database of repetitive DNA families. Nucleic Acids Res. 2016;44:D81-9.

4. Tang W, Mun S, Joshi A, Han K, Liang P. Mobile elements contribute to the uniqueness of human genome with 15,000 human-specific insertions and 14 Mbp sequence increase. DNA Res. 2018;25:521-33.

5. Houck CM, Rinehart FP, Schmid CW. A ubiquitous family of repeated DNA sequences in the human genome. J Mol Biol. 1979;132:289-306.

6. Rubin CM, Houck CM, Deininger PL, Friedmann T, Schmid CW. Partial nucleotide sequence of the 300-nucleotide interspersed repeated human DNA sequences. Nature. 1980;284:372-4.

7. Schmid CW, Jelinek WR. The Alu family of dispersed repetitive sequences. Science. 1982;216:1065-70.

8. Lander ES, Linton LM, Birren B, Nusbaum C, Zody MC, Baldwin J, et al. Initial sequencing and analysis of the human genome. Nature. 2001;409:860-921.

9. Bailey JA, Gu Z, Clark RA, Reinert K, Samonte RV, Schwartz S, et al. Recent segmental duplications in the human genome. Science. 2002;297:1003-7.

10. Sebat J, Lakshmi B, Troge J, Alexander J, Young J, Lundin P, et al. Largescale copy number polymorphism in the human genome. Science. 2004; 305:525-8.

11. Batzer MA, Deininger PL. Alu repeats and human genomic diversity. Nat Rev Genet. 2002;3:370-9.
12. Bennett EA, Keller H, Mills RE, Schmidt S, Moran JV, Weichenrieder O, et al. Active Alu retrotransposons in the human genome. Genome Res. 2008;18: 1875-83.

13. Konkel MK, Walker JA, Hotard AB, Ranck MC, Fontenot CC, Storer J, et al. Sequence analysis and characterization of active human Alu subfamilies based on the 1000 genomes pilot project. Genome Biol Evol. 2015;7:2608-22.

14. Lee J, Kim Y-J, Mun S, Kim H-S, Han K. Identification of human-specific AluS elements through comparative genomics. Gene. 2015;555:208-16.

15. Xing J, Zhang Y, Han K, Salem AH, Sen SK, Huff CD, et al. Mobile elements create structural variation: analysis of a complete human genome. Genome Res. 2009;19:1516-26.

16. Stewart C, Kural D, Strömberg MP, Walker JA, Konkel MK, Stütz AM, et al. A comprehensive map of mobile element insertion polymorphisms in humans. PLoS Genet. 2011;7:e1002236.

17. Deininger PL, Batzer MA. Alu repeats and human disease. Mol Genet Metab. 1999;67:183-93 Available from: http://www.ncbi.nlm.nih.gov/entrez/query. fcgi?cmd=Retrieve\&db=PubMed\&dopt=Citation\&list_uids=10381326.

18. Belancio VP, Hedges DJ, Deininger P. Mammalian non-LTR retrotransposons: For better or worse, in sickness and in health. Genome Res. 2008;18:343-58.

19. Estecio MRH, Gallegos J, Dekmezian M, Lu Y, Liang S, Issa J-PJ. SINE retrotransposons cause epigenetic reprogramming of adjacent gene promoters. Mol Cancer Res 2012;10:1332-1342. Available from: https://doi. org/10.1158/1541-7786.MCR-12-0351

20. Elbarbary RA, Lucas BA, Maquat LE. Retrotransposons as regulators of gene expression. Science. 2016;351(6274):aac7247.

21. Chen LL, Yang L. ALUternative regulation for gene expression. Trends Cell Biol. 2017;27:480-90

22. Solyom S, Kazazian HH. Mobile elements in the human genome: implications for disease. Genome Med. 2012;4:12.

23. Kazazian HH, Moran JV. Mobile DNA in health and disease. N Engl J Med. 2017;377:361-70.

24. Payer LM, Steranka JP, Yang WR, Kryatova M, Medabalimi S, Ardeljan D, et al. Structural variants caused by Alu insertions are associated with risks for many human diseases. Proc Natl Acad Sci U S A. 2017;114:E3984-92.

25. Putku M, Kepp K, Org E, Sõber S, Comas D, Viigimaa M, et al. Novel polymorphic AluYb8 insertion in the WNK1 gene is associated with blood pressure variation in Europeans. Hum Mutat. 2011;32:806-14.

26. Hormozdiari F, Alkan C, Ventura M, Hajirasouliha I, Malig M, Hach F, et al. Alu repeat discovery and characterization within human genomes. Genome Res. 2011:21:840-9.

27. Hormozdiari F, Hajirasouliha I, Dao P, Hach F, Yorukoglu D, Alkan C, et al. Next-generation VariationHunter: combinatorial algorithms for transposon insertion discovery. Bioinformatics. 2010;26:1350-7.

28. Quinlan AR, Clark RA, Sokolova S, Leibowitz ML, Zhang Y, Hurles ME, et al. Genome-wide mapping and assembly of structural variant breakpoints in the mouse genome. Genome Res. 2010;20:623-35.

29. Lee E, Iskow R, Yang L, Gokcumen O, Haseley P, Luquette L, et al. Landscape of somatic retrotransposition in human cancers. Science. 2012;337:967-71.

30. Keane TM, Wong K, Adams DJ. RetroSeq: transposable element discovery from next-generation sequencing data. Bioinformatics. 2013;29:389-90.

31. David M, Mustafa H, Brudno M. Detecting Alu insertions from highthroughput sequencing data. Nucleic Acids Res. 2013;41:e169.

32. Wu J, Lee W-P, Ward A, Walker JA, Konkel MK, Batzer MA, et al. Tangram: comprehensive toolbox for mobile element insertion detection. BMC Genomics. 2014;15:795.

33. Sudmant PH, Rausch T, Gardner EJ, Handsaker RE, Abyzov A, Huddleston J, et al. An integrated map of structural variation in 2,504 human genomes. Nature. 2015;526:75-81.

34. Fiston-Lavier A-S, Barrón MG, Petrov DA, González J. T-lex2: genotyping, frequency estimation and re-annotation of transposable elements using single or pooled next-generation sequencing data. Nucleic Acids Res. 2015; 43:e22.

35. Santander CG, Gambron P, Marchi E, Karamitros T, Katzourakis A, Magiorkinis G. STEAK: A specific tool for transposable elements and retrovirus detection in high-throughput sequencing data. Virus Evol. 2017;3:vex023.

36. Witherspoon DJ, Zhang Y, Xing J, Watkins WS, Ha H, Batzer MA, et al. Mobile element scanning (ME-scan) identifies thousands of novel Alu insertions in diverse human populations. Genome Res. 2013;23:1170-81.

37. Wildschutte $\mathrm{JH}$, Baron A, Diroff NM, Kidd JM. Discovery and characterization of Alu repeat sequences via precise local read assembly. Nucleic Acids Res. 2015:43:10292-307. 
38. Wang J, Song L, Grover D, Azrak S, Batzer MA, Liang P. dbRIP: a highly integrated database of retrotransposon insertion polymorphisms in humans. Hum Mutat. 2006;27:323-9.

39. Pajuste F-D, Kaplinski L, Möls M, Puurand T, Lepamets M, Remm M. FastGT: an alignment-free method for calling common SNVs directly from raw sequencing reads. Sci Rep. 2017;7:2537.

40. Mitt M, Kals M, Pärn K, Gabriel SB, Lander ES, Palotie A, et al. Improved imputation accuracy of rare and low-frequency variants using populationspecific high-coverage WGS-based imputation reference panel. Eur J Hum Genet. 2017;25:869-76.

41. Li H, Handsaker B, Wysoker A, Fennell T, Ruan J, Homer N, et al. The sequence alignment/map format and SAMtools. Bioinformatics. 2009;25: 2078-9.

42. Hulsen T, de Vlieg J, Alkema W. BioVenn - a web application for the comparison and visualization of biological lists using area-proportional Venn diagrams. BMC Genomics. 2008;9:488.

43. Yu Q, Zhang W, Zhang X, Zeng Y, Wang Y, Wang Y, et al. Population-wide sampling of retrotransposon insertion polymorphisms using deep sequencing and efficient detection. Gigascience. 2017;6:1-11.

44. Untergasser A, Cutcutache I, Koressaar T, Ye J, Faircloth BC, Remm M, et al. Primer3-new capabilities and interfaces. Nucleic Acids Res. 2012;40:e115.

45. Koressaar T, Remm M. Enhancements and modifications of primer design program Primer3. Bioinformatics. 2007;23:1289-91.

46. Andreson R, Puurand T, Remm M. SNPmasker: automatic masking of SNPS and repeats across eukaryotic genomes. Nucleic Acids Res. 2006;34:W651-5.

47. Köressaar T, Lepamets M, Kaplinski L, Raime K, Andreson R, Remm M. Primer3-masker: integrating masking of template sequence with primer design software. Bioinformatics. 2018;34:1937-8.

\section{Publisher's Note}

Springer Nature remains neutral with regard to jurisdictional claims in published maps and institutional affiliations.

Ready to submit your research? Choose BMC and benefit from:

- fast, convenient online submission

- thorough peer review by experienced researchers in your field

- rapid publication on acceptance

- support for research data, including large and complex data types

- gold Open Access which fosters wider collaboration and increased citations

- maximum visibility for your research: over $100 \mathrm{M}$ website views per year

At $\mathrm{BMC}$, research is always in progress.

Learn more biomedcentral.com/submissions 